\title{
Hybrid Intrusion Detection System Using K-means and J-48
}

\author{
Manvir Singh ${ }^{1}$ and Jatinder Singh $\mathrm{Bal}^{2}$ \\ Research scholar ${ }^{1}$, Professor, DAV University, Jalandhar ${ }^{2}$ \\ Dept. of Computer science and Engineering \\ manvirdav@gmail.com ${ }^{1}$, bal_jatinder@rediffmail.com ${ }^{2}$
}

\begin{abstract}
Security attacks through internet are increased continuously in recent years therefore the Information security is globally very serious problem at the present time. The importance of protecting networks is most important. The need of intrusion detection system has been brought out. In this work a hybrid intrusion system based on data mining techniques using $K$ means and $\mathrm{J} 48$ is proposed. In this paper work hybrid intrusion detection system is proposed first Misuse based detection model is build using K-means clustering algorithm then based upon the output of misuse detection phase Anomaly based intrusion detection model is build using improved J-48 classification algorithm in this way the combine output of these two phases attains very higher detection accuracy. For training and testing of hybrid intrusion detection model famous NSL-kdd dataset is used.
\end{abstract}

Keywords: Intrusion detection, Misuse detection, Anomaly detection, NSL-kdd dataset.

\section{Introduction}

Information security problems are well known. However many studies has been done to secure the information systems from intrusions. But there is still an open research area to secure the network information systems (both wired and wireless). With advancements in network communication technologies, Internet has been undergoing vast growing in network traffic also followed by an increasing number of system and network intrusions such as Denial of Service (DoS) attacks, worms, virus exploits and many more. Therefore, detecting and preventing these threats are complex tasks for network operators/administrators to ensure that the Internet resources remain available. Because large traffic must be able to travel efficiently, quickly and accurately identifying anomalies or intrusions in network traffic is important, therefore it requires development of good detection techniques. There is an urgent need to intelligent intrusion detection systems (IDSs) to detect novel intrusions automatically [2].

Intrusions are patterns of interest to network defenders, who want to extract them from vast amount of network traffic data. To overcome this Data mining techniques have been popular in extracting these harmful patterns from large volumes of data in recent years. Data mining is used in many useful application areas, business world, medical sciences, physical sciences engineering and many more. Many studies have been performed in applying data mining techniques to network traffic intrusions detection, but the methods still have limitations for use in real environments. In our work we use two famous data mining techniques K-means for clustering and J-48 for classification.

There are two methodologies used to detect intrusions:

a.) Misuse based intrusion detection and b.)Anomaly based intrusion detection system. 
Misuse based intrusion detection also known as signature based intrusion detection method because it works same as like antivirus. It detects intrusions by matching signatures of patterns that is already stored in database. Anomaly based intrusion detection also known as behavior based intrusion detection because it detects intrusion by checking the behavior of intrusions patterns. Deviation of normal is considered as an intrusion.

Rest of paper is organized as follows Section 2 describe brief introduction of intrusion detection system. Section 3 describes work related to intrusion detection. Section 4 presents proposed work in detail. Section 5 presents results and discussion work in detail. Section 6 presents conclusion and future work.

\section{Intrusion Detection System}

An Intrusion Detection System is an application used for monitoring the network and protecting it from the intruder [15].Intrusion detection system is an active research area but intrusion detection undergoes several problems with improvements in security the risks of intrusion is still a matter of concern. Intrusions are an unwanted access to the system by breaking the security. In other words we describe intrusions same as an attacks. Intrusions are the actions that break the security wall of the system and the intrusion detection system is the system which is designed and employed to detect these intrusions. In general "An intrusion detection system is a hardware device or software application that watches network or system activities for watching malicious activities or policies and raises the security alarm to system administrator".

According to SANS institute intrusion detection refers to all processes used in discovering unauthorized use of network or computer devices [17].

In simple terms Intrusion Detection System (IDS) watches computer system network traffic, then analyzes the traffic to identify and detect possible security breaches and raises alerts to system administrator. Intrusion detection means exposing/catching unauthorized use of system or network. Intrusion Detection Systems are implemented either in software or hardware in order to detect malicious activities Intrusion Detection System is also well known as second layer of defense after Firewall. The Firewall were not designed to handle Network and Application layer attacks such as Denial of service attacks, worms, viruses and Trojans. Intrusion Detection generally operates behind firewall.

IDS usually comprises of 3 components:

Data Collection: Responsible for data collection, preprocessing the data etc. and send data to detection phase

Analysis: Received data is analyzed and analyzed data sent to response component. Analysis component is also known as detection component phase.

Response component: Response component responds immediately to detected anomalies and sent alarm to system administrator.

\subsection{Classification of Intrusion detection system}

\section{a.) Host based IDS (HBIS)}

Host based Intrusion Detection System resides on individual host or network. Host based IDS monitors inbound and outgoing packets from the network device and will alert the network administrator if there is any doubtful/suspicious activity is detected. Host based IDS generally involves a security agent installed on each system, monitoring and alerting on local 
operating system. The installed agent uses a combination of signatures, rules, and heuristics from database to identify and minimize unauthorized activities.

\section{b.) Network based IDS (NBIS)}

Network based Intrusion Detection System analyze, evaluate and monitors network traffic to and from all devices connected on network. It performs a complete analysis of traffic passing on entire network and matches the traffic characteristics with database of already stored signatures/characteristics. Once the signatures/characteristics of network traffic are matched with anomalous traffic signatures/characteristics it generates security message/alarm to the system administrator.

\subsection{Intrusion Detection Methodologies}

In order to determine how intrusion detection system works to identify intrusions therefore there are mainly two approaches available for use in Intrusion Detection System i.e. Misuse based and Anomaly based detection approaches.



\section{Misuse based intrusion detection approach}

Also known as Signature based Intrusion Detection system. Signature based intrusion detection approach works similar to the antivirus system. It matches signature/patterns (well known string matching) that are already store in database to detect attacks.

Signature based intrusion detection system has many benefits it has very high detection rate of attacks due to already stored signature in database it match signature and generate security messages/alarms if any suspicious signature found to the system administrator.

Signature based IDS also has very low false positive rate .It has high true positive rate. Signature based intrusion detection system one biggest disadvantage it has very low detection rate for Zero day attacks i.e. newly created attacks. They have ability to detect known attacks with very low error rate [1].

\section{Anomaly based intrusion detection approach}

Anomaly based intrusion detection also known as Behavior based Intrusion detection. Anomaly detection model analyze normal traffic. Anomaly detection model is based on the hypothesis/assumption that attacker/malicious behavior different from that of normal traffic behavior [1]. Anomaly based intrusion detection system classify traffic as an attack if the characteristics/patterns of traffic are far away from normal traffic characteristics/ patterns.

In simple words first anomaly detection model creates a baseline profile of normal traffic activities this process is known as training part of the system. Then after any new traffic activity that deviating from normal traffic is considering as an anomalies. High false positive rate makes the detection system useless [7]. 
Biggest advantage of Anomaly based IDS detection model over Misuse based IDS detection model is that they are very effective for newly created attacks. The one and only one disadvantage of Anomaly detection model is that they are not effective as misuse based intrusion detection model in detection rate. They produces high false positive rate. False positive means they raise alarm for unsuccessful attack attempt.

\section{Related Work}

Kim et al. [1] proposed a novel hybrid intrusion detection model integrating anomaly detection with misuse detection first a misuse detection model is built the data is decomposed into smaller subsets then based upon the output then multiple one class support vector models is created for each decomposed set to evaluate the performance. In this technique NSL Kdd 99 data set is used for conducting experiments.

2Elbasiony et al. [2] proposed a hybrid intrusion detection framework based on random forest and weighted $\mathrm{k}$ means uses the concept of data mining and clustering techniques, in proposed technique random forest classification algorithm is used to build intrusion patterns to detect intrusions and $\mathrm{k}$ means clustering algorithm is used to detect novel intrusions by clustering the network connections. In this technique kdd99 data set is used for evaluation.

Chandrasekhar et al. [3] proposed a novel intrusion detection technique by using $\mathrm{k}$ means, fuzzy neural network and SVM classifiers. Firstly $\mathrm{k}$ means clustering is used to generate different training subsets then based upon obtained subset different neuro fuzzy models are trained at the end classification using SVM is done to detect intrusion or not. In this work kdd99 data set is used for experiments.

Feng et al. [4] proposed a novel technique by combining Ant colony with SVM. The experiments are conducted on kdd cup 99 dataset. In this paper, a new algorithm combining support vectors with ant colony (CSVAC) for generating classifiers with clustering, and applied it to the intrusion detection problem.

Mitchell et al. [5] surveys various Intrusion Detection Techniques in Wireless network applications. The approach is to classify existing contemporary wireless intrusion detection system (IDS) techniques based on target wireless network, detection technique, collection process, and trust model and analysis technique. They summarize pros and cons of the same or different types of concerns and considerations for wireless intrusion detection with respect to specific attributes of target wireless networks including wireless local area networks (WLANs), wireless personal area networks (WPANs), wireless sensor networks (WSNs), ad hoc networks, mobile telephony, wireless mesh networks (WMNs) and cyber physical systems (CPSs). They summarize the most and least studied wireless IDS techniques in the literature, identify Research gaps, and analyze the rationale for the degree of their treatment.

Barbara et al. [6] proposed Audit Data Analysis and Mining method where anomaly detection is followed by misuse detection .They uses a combination of association rule mining and a classification method to detect attacks. First Anomaly detection model is build using association rule mining locates suspicious traffic connections and passes connection to misuse detection model then the misuse detection model specifies the connections as normal or suspicious.

Zhang et al. [7] used misuse detection model followed by anomaly detection model to design a hybrid intrusion detection model by using random forests technique. The misuse detection model is use first to detect known attacks with low false positive rate and operate faster than anomaly detection. The anomaly detection model is only applied to remaining part of connections. Then results from misuse and anomaly model are combined. The kdd99 data set is used to evaluate perform evaluations. 
Tavallaee et al. [8] perform a detailed analysis of the KDD CUP 99 data set. They conducted a statically analysis of kdd 99 data set and found important issues which highly affect the performance of evaluated systems. They solved the issues by proposing a new modified data set NSL kdd data set.

Siddiqui et al. [9] perform analysis of 10\% of KDD cup'99 training dataset based on intrusion detection. They focused on establishing a relationship between the attack types and the protocol used by the hackers, using clustered data. Analysis of data is performed using kmeans clustering; they used the Oracle $10 \mathrm{~g}$ data miner as a tool for the analysis of dataset and build 1000 clusters to segment the 494,020 records. The investigation revealed many interesting results about the protocols and attack types preferred by the hackers for intruding the networks.

Chaturvedi et al. [10] proposed a technique on combining decision tree and Random forest used in data mining. For experiments and evaluation of results they use famous kdd 99 data set available for researchers for evaluations. Attacks are detected using decision tree C4.5 then apply Random forest technique to improve the results of C4.5 algorithm. They have also used PCA (Principal Component analysis) to reduce the dimensionality of datasets required to detect attacks. They compare the results with Naives Bayes classification algorithm. They classify C4.5 and Naives bayes to show which one is best to classify Attack.

\section{Proposed Work}

\subsection{Introduction}

The rapid increase of online systems has changed the landscape of network security. The traditional way of protecting networks with firewalls and encryption based softwares is no longer effective. There are number of new techniques available for detecting the intruders/anomalies but there is still an issue of developing and designing effective intrusion detection system for network information systems to overcome the problems of false positives and false negatives. Due to increase in number of attacks/anomalies/intrusions it is compulsory and first step of securing systems is to design a more securing and effective intrusion detection system that can detect and respond intruders as early as possible.

\subsection{Proposed Technique}

A hybrid intrusion detection system based on data mining techniques is proposed. NSLkdd dataset is used for evaluation of results and training and testing of proposed hybrid intrusion detection system. In this system first misuse based intrusion detection model (also known signature based) is designed using data mining k-means algorithm which is used to make clusters with training dataset. Misuse detection phase is our training part of intrusion detection. Then anomaly detection phase is built based on output of misuse detection model using improved J48 classification algorithm which is also known as decision tree algorithm to classify instances into two categories i.e. anomaly and normal. The anomaly detection model is tested using testing dataset to detect anomalies and normal data.

\subsubsection{Dataset used for training and testing}

In this work NSL-kdd dataset is used for training and testing of our proposed intrusion detection system. NSL-kdd dataset is modified version (contains selected records of kdd cup 1999 dataset) of famous dataset KDD cup1999. The kdd cup 99 dataset was created by 
preprocessing the tcp dump of 1998 DARPA IDS evaluation dataset created by Lincoln lab. In kdd dataset there is number of problems for use in intrusion detection system.

The first problem of kdd cup 1999 is that it contains large number of redundant records in training and testing datasets. These redundant records will cause learning algorithms to be biased towards the more frequent records and these redundant records will cause evaluation results to be biased by methods which have better detection rates on frequent record [8].

NSL-kdd dataset is modified version of kdd cup 1999 dataset which does not contains redundant records of same kind. NSL-kdd dataset contains total of 125974 records in training dataset and 22545 records in testing dataset. Both train and testing datasets of NSL-kdd contains attacks and normal data connections. The attacks present in training data set not included in testing data set due to measuring the performance of intrusion detection systems i.e. testing data includes specific attacks types that is not in training dataset .

Benefits of NSL - kdd dataset over kdd cup 1999

1) It does not include any redundant records in the train set, so the classifiers will not have to be biased towards more frequent records.

2) There is no duplicate records in the NSL-kdd test dataset therefore the performance of the learners are not biased by the methods which have better detection rates on the frequent records.

3) The number of selected records from each difficulty level group is inversely proportional to the percentage of records in the original KDD data set. As a result, the classification rates of distinct machine learning methods vary in a wider range, which makes it more efficient to have an accurate evaluation of different learning techniques.

4) The number of records in the train and test sets is reasonable, which makes it affordable to run the experiments on the complete set without the need to randomly select a small portion. Consequently, evaluation results of different research works will be consistent and comparable

\subsection{Methodology}

a) Collect raw data from NSL-kdd dataset which is modified version of famous kdd cup 1999 network traffic dataset. NSL-kdd contains non redundant records

b) Apply CFS feature selection algorithm. CFS is correlation based feature selection. Feature selection method try to pick up subsets of features that are relevant to the target concept. CFS quickly identifies and screens irrelevant, redundant, and noisy features, and identifies a relevant feature that does not strongly depend on other features. CFS typically eliminated well over half the features. In simple words CFS identifies only best features that are useful for improving performance of IDS.

d) Design Misuse detection model with famous data mining $\mathrm{k}$ means clustering algorithm. In misuse detection model (also called signature based detection) we use the training dataset of NSL-kddtrain.csv file which is used to train our misuse detection phase. This phase of misuse detection model is for training of our proposed system further this misuse detection model output gives input (as training) to next model known as Anomaly detection model.

Training process of Misuse detection model:

Step1: Prepare a training data set consisting of normal data and known attack data.

Step2: Build a misuse detection model based on the training data set.

Step3: Decompose the normal training data into clusters using k means clustering algorithm. 
K-means clustering algorithm is an unsupervised method of learning [3]. The basic terminology used in K-means clustering algorithm is that it partitions $\mathrm{n}$ observations that are in data items into $\mathrm{k}$ clusters in which each data item belongs to the cluster having the nearest mean. According to this, in the beginning we will determine " $\mathrm{K}$ " that is, the number of clusters and we will assume the centroid or center of these clusters using the distance between two objects and taking their mean value.

e) Then Anomaly detection phase (also known as behavior based intrusion detection) is designed based on output of misuse detection phase. The output of misuse detection model is used as training data to anomaly detection model. The anomaly detection model is build using improved J48 classification algorithm.

Testing process of Anomaly detection:

Step1: Collecting the clustered training data from misuse detection model as input to anomaly detecting model.

Step2: Classify the dataset as normal and anomalous by using improved J48 classification algorithm.

The J48 classification algorithm is widely used classification algorithm in data mining. The J48 consists of nodes, edges, and leaves. A J48 node has its corresponding data set this specifies the attribute to best divide the data set into its classes. Each node has several edges that specify possible values or value ranges of the selected attributes on the node.

The J48 algorithm recursively visits each decision node, selecting the optimal split, until no further splits are possible. The basic steps of $\mathrm{J} 48$ algorithm for growing a decision tree are given below:

The data set of the node is divided into subsets according to the specifications of the edges, and the J48 creates a child node for each data subset and repeats the dividing process. When the node satisfies the stopping rules because it contains homogeneous data sets or no future distinguishing attributes can be determined, the $\mathrm{J} 48$ terminates the dividing process and the node is labeled as following the class label of the data set. This labeled node is called a leaf node. In this way, the J48 recursively partitions the training data set, which creates a tree-like structure [1].

In our paper we use improved J48 classification algorithm. The basic J48 apply on whole dataset which may reduce some performance accuracy and some time consuming but the improved J48 classification algorithm recursively partitions the dataset into just like small trees until and then improved J48 can apply on those small trees also called small bags (apply small parts of dataset) then combine the results to give output this can improve detection accuracy and performance of intrusion detection..

f) Then in last we measure the performance of proposed hybrid intrusion detection system in terms of detection accuracy rate, false positive rate, and compare the results of our proposed intrusion detection system with other already proposed systems [1], [2], [7] 


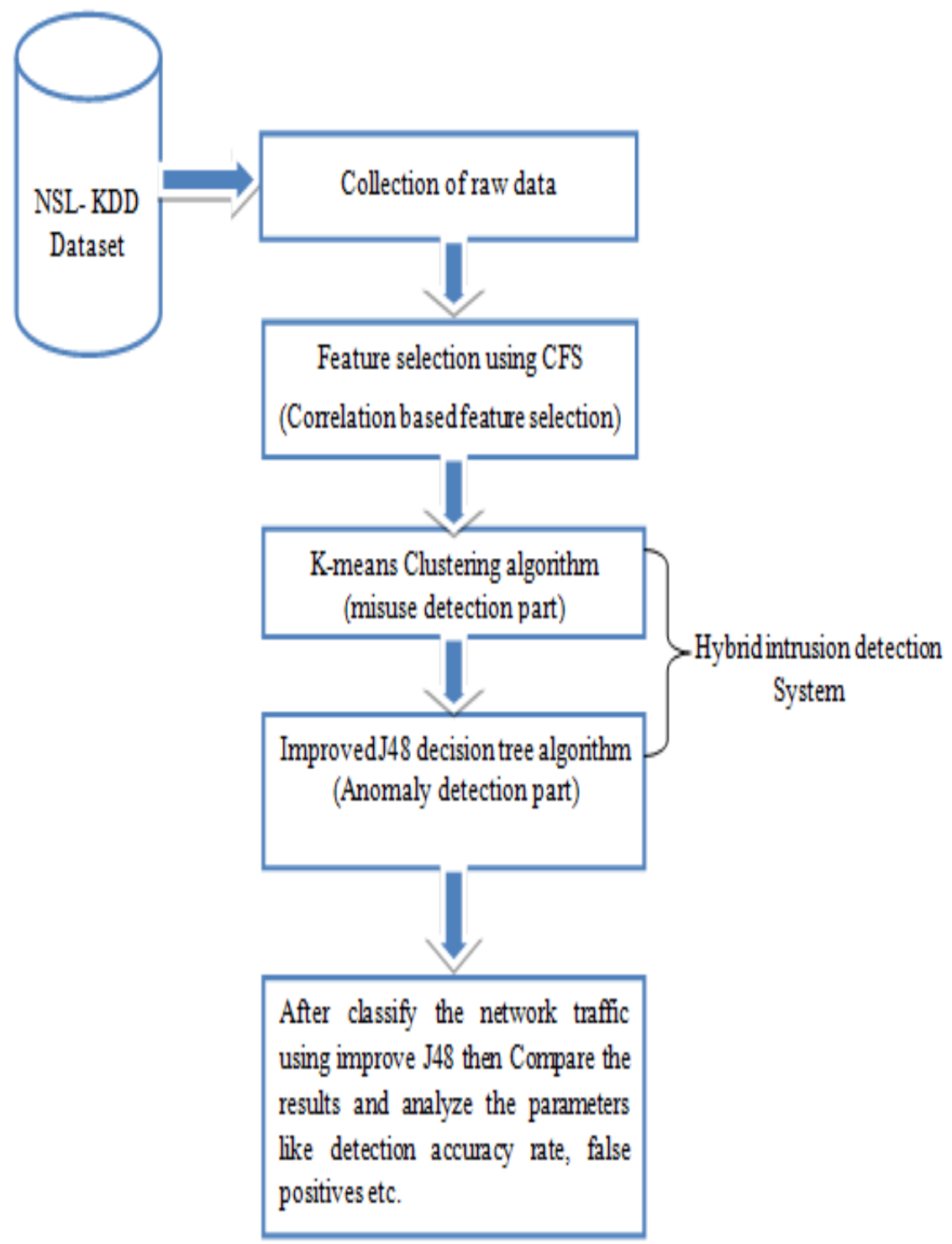

Figure 4.3.1 Flow Chart of Methodology

\section{Results and Discussion}

Our proposed intrusion detection model is implemented in Netbeans IDE 7.4 Platform (Java) and with help of weka tool. In the proposed hybrid intrusion detection system the training and testing of system using NSL-kdd dataset with 3 different subsets is performed i.e. (1.) $10 \%$ of training and testing dataset and (2.) $20 \%$ of training and testing dataset (3.) full dataset respectively.

First we compare our results with already developed intrusion detection systems with $10 \%$ dataset only and then in addition separately 3 dataset subsets results is shown i.e. $10 \%$, $20 \%$, full dataset respectively.

Firstly, the table shows the comparison of our proposed intrusion detection system with other systems with $10 \%$ train and test dataset. 
Table 5.1 Comparison of Results with other Proposed Systems

\begin{tabular}{|l|c|c|c|}
\hline Approach & Dataset & Detection rate \% & False positive rate\% \\
\hline $\begin{array}{l}\text { Method introduced } \\
\text { in [1] }\end{array}$ & $10 \%$ NSL kdd & 99 & 1 \\
\hline $\begin{array}{l}\text { Method introduced } \\
\text { in [2] }\end{array}$ & $10 \%$ kdd 1999 & 98.3 & 1.2 \\
\hline $\begin{array}{l}\text { Method introduced } \\
\text { in [7] }\end{array}$ & $10 \%$ kdd 1999 & 94.7 & 2 \\
\hline $\begin{array}{l}\text { Proposed system } \\
\text { 10\% NSL-kdd }\end{array}$ & 99.9 & 0.78 \\
\hline
\end{tabular}

Therefore, the false positive rate is FP/FP+TP. where FP is number of false positives, and TN is the number of true negatives.



Figure 5.1 Results Comparison Chart

Testing of the proposed system using NSL-kdd dataset with the three different subsets

1.) $10 \%$ of the training and testing dataset and 2.) $20 \%$ of training and testing dataset 3.) full dataset respectively. 
Table 5.2 Subset Wise Results

\begin{tabular}{|l|c|c|c|}
\hline \multirow{2}{*}{$\begin{array}{l}\text { Proposed Hybrid } \\
\text { IDS system }\end{array}$} & $10 \%$ NSL-kdd & 99.9 & False positive rate \\
\cline { 2 - 4 } & & & 0.78 \\
(Misuse +Anomaly) & $20 \%$ NSL-kdd & 99.5 & 0.89 \\
\cline { 2 - 4 } & Full & 99 & 1 \\
\hline
\end{tabular}

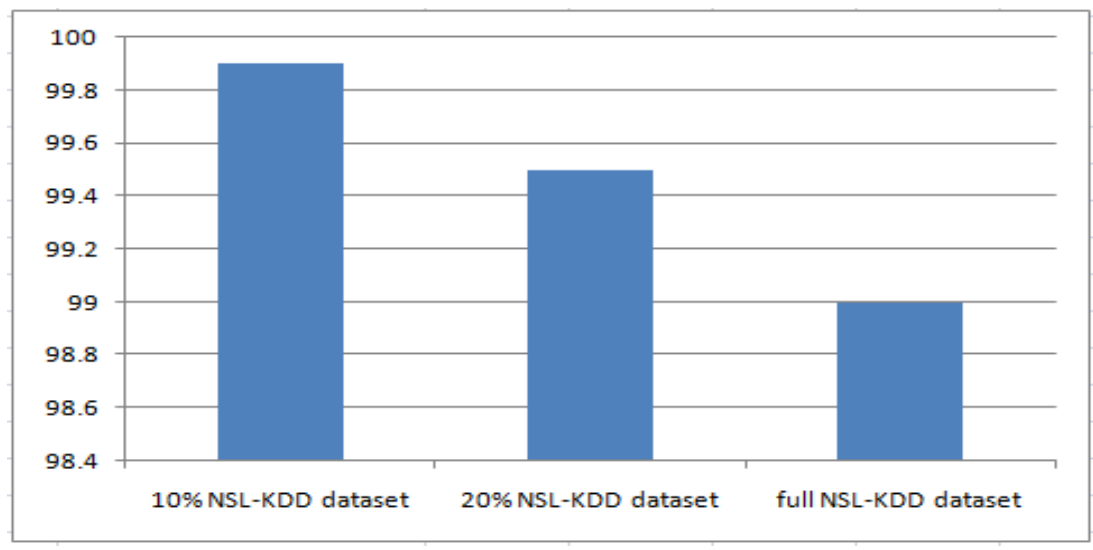

Figure 5.2 Subset Wise Results Chart

\section{Conclusion and Future Work}

\section{Conclusion}

We propose that we can develop a hybrid intrusion model by using data mining techniques. First we develop misuse detection model using K-means clustering algorithm this phase is our training phase of intrusion detection system. Then the clustered data is then passed to testing phase known as anomaly detection phase in which clustered at is passed as an input to improved J-48 classification algorithm. For training and testing of our proposed model we used NSL-kdd dataset which is famous dataset and improved version of KDD 199 cup dataset.

On the basis of theoretical and experimental simulation investigation reported in this work, the followings conclusions have been drawn.

a) Proposed hybrid intrusion detection system has better detection accuracy than other traditional intrusion detection systems.

b) Our proposed system achieve detection rate of $99.9 \%$ (on $10 \%$ dataset) with very low false positives and false negatives and compared with [1], [2], [7]. 


\section{Future Work}

a) Despite being well-investigated fields, the topics covered in this thesis are far from being dead-ends.

b) It is possibility to reduce average training and testing time of datasets used for testing intrusion detection system.

\section{References}

[1] G. Kim, S. Lee, and S. Kim, "A novel hybrid intrusion detection method integrating anomaly detection with misuse detection", Expert System Applications, 2014.

[2] R. M. Elbasiony, E. a. Sallam, T. E. Eltobely, and M. M. Fahmy, "A hybrid network intrusion detection framework based on random forests and weighted k-means”, Ain Shams Eng. Journal , 2013.

[3] A. M. Chandrasekhar and K. Raghuveer, "Intrusion detection technique by using k-means, fuzzy neural network and SVM classifiers", 2013 Int. Conf. Comput. Commun. Informatics,2013.

[4] W. Feng, Q. Zhang, G. Hu, and J. X. Huang, "Mining network data for intrusion detection through combining SVMs with ant colony networks", Futur. Gener. Computer System, 2014.

[5] R. Mitchell and I.-R. Chen, "A survey of intrusion detection in wireless network applications", Comput. Commun., 2014.

[6] D. Barbará, J. Couto, S. Jajodia, L. Popyack, and N. Wu, "ADAM: Detecting Intrusions by Data Mining", Proc. IEEE Work. Inf. Assur. Secur., no. June, 2001.

[7] J. Zhang and M. Zulkernine, "A hybrid network intrusion detection technique using random forests", First Int. Conf. Availability, Reliab. Secur., 2006.

[8] M. Tavallaee, E. Bagheri, W. Lu, and A. a. Ghorbani, "A detailed analysis of the KDD CUP 99 data set", IEEE Symp. Comput. Intell. Secur. Def. Appl. CISDA 2009

[9] M. K. Siddiqui and S. Naahid, "Analysis of KDD CUP 99 Dataset using Clustering based Data Mining", Int. J. Database Theory Appl., vol. 6, no. 5, pp. 23-34, 2013.

[10] S. K. Chaturvedi, "Attack Detection over Network based on C45 \& RF Algorithms", International journal of attacks and security, vol. 15, no. 9, pp. 68-73, 2015.

[11] Poongothai .t and Duraiswamy .k, "Intrusion Detection in Mobile Ad-Hoc Networks using Machine Learning Approach”, ICICES2014 - S.A. Engineering College, Chennai, Tamil Nadu, India , no. 978, pp. 48, 2014.

[12] S. Uyyala, "Anomaly based Intrusion detection of Packet Dropping Attacks in Mobile Ad-hoc Networks", International Conference on Control, Instrumentation, Communication and Computational Technologies, 2014.

[13] K. Wu, W. Zhang, and W. Zhu, "A Study on the Application of Intrusion Detection Technology to WLAN", IEEE 3rd Int. Conf. Commun. Softw. Networks, 2011.

[14] S. V. Shirbhate, S. S. Sherekar, and V. M. Thakare, "A Novel Framework of Dynamic Learning Based Intrusion Detection Approach in MANET", 2015 Int. Conf. Comput. Commun. Control Autom.,2015.

[15] S. Vijayarani and M. Sylviaa, "Intrusion Detection System", Int. J. f Secur. Priv. Trust Managment, 2015

[16] E. Amiri, H. Keshavarz, H. Heidari, E. Mohamadi, and H. Moradzadeh, "Intrusion Detection Systems in MANET: A Review", 2014.

[17] https://www.sans.org/ 
International Journal of Security and Its Application Vol.11, No.1 (2017) 